\title{
Gridless retarding potential analyzer for use in very-low- energy charged particle detection
}

\author{
T. W. Shyn, W. E. Sharp, and P. B. Hays \\ Department of Atmospheric and Oceanic Science, Space Physics Research Laboratory, University of Michigan, Ann Arbor, Michigan 48109 \\ (Received 25 February 1976; in final form, 6 May 1976)
}

\begin{abstract}
The theory of the hyperbolic retarding potential analyzer in the electrostatic mode is developed in detail and verified in the laboratory. A monoenergetic electron beam is used for the laboratory investigation. The analyzer (acronym HARP) has advantages over other conventional electrostatic analyzers; among them are less contact potential influence and high throughput because of the symmetry shape of the analyzer. The most useful application of the HARP is in detecting low-energy charged particles. A sample of low-energy particle data obtained in the earth's ionosphere is given.
\end{abstract}

\section{INTRODUCTION}

Low-energy electrons play a fundamental role in the energetics of planetary atmospheres. These electrons are produced as a result of ionizing processes in the atmospheric gases. After creation, the electrons lose their energy, principally in inelastic collisions (leading to excited molecules and atoms) and elastic collisions (leading to enhanced thermal electron temperatures), and eventually become a part of the thermal electron population. Knowledge of the energy distribution of these electrons is desirable in order to determine the energy budget of the atmosphere.

Many types of instruments have been utilized on space probes to find this distribution. Various types of Langmuir probes ${ }^{1}$ and retarding potential analyzers ${ }^{2}$ measure the integral distributions, and cylindrical ${ }^{3}$ and spherical ${ }^{4}$ electrostatic analyzers are used to measure the differential distribution. The ratio of the deflecting potential to the deflected particle energy is of fundamental importance for measuring low-energy electrons. Surface contact potentials become a problem below $1 \mathrm{~V}$, and consequently, with small ratios, the particles may have their trajectories considerably distorted. Conventional analyzers used on spacecraft (spherical and cylindrical) have a ratio that is generally less than unity (order of $0.5-0.1$ ). To obtain a unity ratio requires that the plate separations be approximately equal to their radii. This introduces serious field distortions from the edges as well as a reduction in particle focusing properties.

This paper describes in detail an instrument, a gridless analyzer, which, although not a new kind, has certain unique properties when used on spacecraft to measure low-energy charged particles. The first property is that the deflecting potential to particle energy ratio is never less than unity and can be made much larger than unity. A second property is that it minimizes contact potential problems common to most energy analyzers. The instrument is a gridless retarding potential analyzer with a hyperbolic field. It is given the acronym HARP. A brief discussion of this instrument has been given elsewhere."

The basic theory of the instrument has been developed previously by several authors ${ }^{6-8}$ in terms of an electron lens and an energy filter for paraxial rays (perpendicular to the lens). Also, the instrument has been discussed by other authors ${ }^{9,10}$ in the context of an rf-driven mass spectrometer. General reviews on the retarding potential analyzers can also be found elsewhere. ${ }^{11,12}$ This paper discusses an essentially identical instrument in terms of an electrostatic energy analyzer. Here, however, the case of meridional rays and nonparaxial incidence with no $r f$ field is treated. The HARP is considered a lenslike device for selectively passing charged particles of various energies.

Section II of the paper discusses the theoretical formulation of particle trajectories in the applied field. In Sec. III, laboratory studies are considered in light of the theory, and in Sec. IV the use of the instrument as a plasma detector in the earth's ionosphere is discussed. It is shown that the HARP is an excellent instrument for measuring low-energy plasma distributions from a few tenths to hundreds of electron volts.

\section{THEORY}

\section{A. Description of the HARP}

The geometry of the HARP consists, essentially, of three solid electrodes in the form of a body of revolution as shown in Fig. 1. The three electrodes consist of a center hyperbolic electrode and two hyperbolic end-cap electrodes (the name "end-cap electrode" comes from the fact that the upper and lower electrodes close the system).

The HARP has a rotationally symmetric quadrupole field, $\phi(\rho, Z)$, produced by the three electrodes and performs the energy selection of the charged particle as follows: For a fixed potential field, particles with the appropriate initial values of energy, position, and direc- 


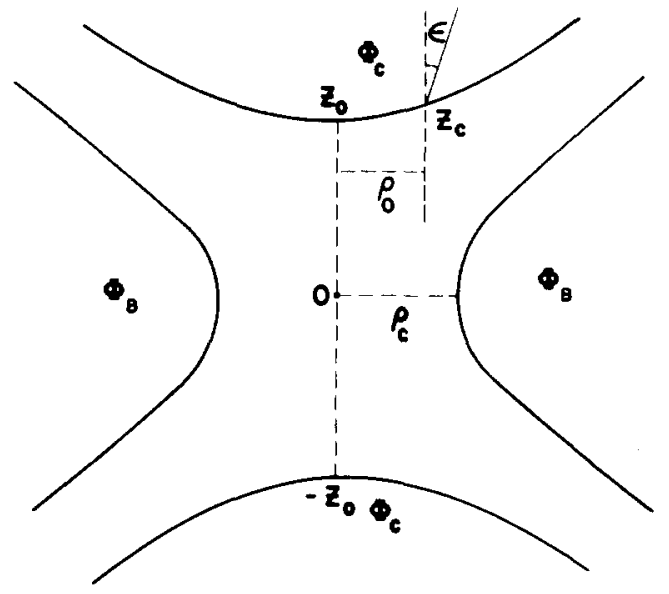

Fig. 1. Geometry of the hyperbolic analyzer. The geometry has a rotational symmetry about the $Z$ axis. $\phi_{b}$ is a potential applied on the center hyperbolic electrode a distance $\rho_{r}$. from origin, $\phi_{c}$, the potential applied on the two (upper and lower) hyperbolic end-caps a distance $Z_{0}$ from the origin, and $\rho_{0}$ and $\epsilon$ are the initial position and angle of the injected electron. $Z_{r}$ is the $Z$ value on the end-caps.

tion are transmitted; the rest are not. The potential field in the cylindrical coordinate system is given by

$$
\phi(\rho, Z)=\left(\phi_{c}-\phi_{B}\right)\left[\frac{\rho_{c}{ }^{2}+2 Z^{2}-\rho^{2}}{\rho_{c}{ }^{2}+2 Z_{0}{ }^{2}}\right]+\phi_{B} .
$$

$\phi_{B}$ is a static potential applied to the center hyperbolic electrode, whose geometry is defined by the relation

$$
\rho=\left(2 Z^{2}+\rho_{\mathrm{r}}{ }^{2}\right)^{1 / 2} .
$$

$\phi_{c}$ is the potential on the two end-cap electrodes whose geometry satisfies the relation

$$
Z_{c}= \pm\left(Z_{0}^{2}+\rho^{2} / 2\right)^{1 / 2} .
$$

Now $\rho_{c}$ in Eqs. (1) and (2) is the distance from the center of the cavity to the hyperbolic electrode, and $Z_{0}$ in Eqs. (1) and (3) is the axial distance to the end-cap electrode from the center of the cavity.

A major convenience of this geometry is that the equations of motion are two-dimensional, separable, and can be solved in analytic form.

\section{B. Trajectories of a charged particle}

The classical Lagrangian $L$ of a charged particle (mass $m$ and charge $q$ ) in the potential field $\phi(\rho, Z)$ in the cylindrical coordinate system is given by

$$
L=(m / 2)\left(\dot{\rho}^{2}+\rho^{2} \dot{\theta}^{2}+\dot{Z}^{2}\right)-q \phi(\rho, Z),
$$

where the dot indicates the time derivative.

As shown in Eq. (4), $\theta$ is cylic and $m \rho^{2} \dot{\theta}$, which is the angular momentum along the $Z$ axis, is a constant of motion. If the initial angular velocity is chosen to be zero (meridional case) as an initial condition of the problem, the problem is reduced to a two-dimensional one as shown below (a more general solution can be found elsewhere $\left.{ }^{7,8}\right)$ :

$$
\frac{d \dot{\rho}}{d t}=-\omega_{D}^{2} \rho
$$

$$
\frac{d \dot{Z}}{d t}=+2 \omega_{l}^{2} Z
$$

where

$$
\left.\omega_{l}\right)^{2} \equiv 2 q\left(\phi_{B}-\phi_{c}\right) /\left(\rho_{c^{2}}{ }^{2}+2 Z_{0}{ }^{2}\right) m .
$$

The negative $2: 1$ ratio between the variation with distance of the field in the two directions of Eqs. (5) and (6) shows that, when the electric field is accelerating a charged particle toward the origin along the $Z$ axis $(+$ or -$)$, it must be decelerating toward the origin along the $\rho$ axis and vice versa.

It is convenient to refer to the mode when the electric field accelerates the particle toward the origin along the $\%$ axis as "the accelerating mode" and to use the term "retarding mode" when the particle decelerates loward the origin. As was noted in the previous section, the charged particle entering from the top-cap electrode will be considered. This will not cause any loss of generality. The retarding mode will be considered first. followed by the accelerating mode.

\section{Retarding mode}

This is the mode in which the energy selection is performed. The coefficient $\omega_{l}{ }^{2}$ in Eqs. (5) and (6) is positive. which implies that $\phi_{B s}<\phi_{c}$, for $q<0$ (electrons and negative ions) or $\phi_{B}>\phi_{C}$ for $q>0$ (positive ions). The solutions of Eqs. (5) and (6) are given by, respectively,

$\rho(t)=\left(\dot{\rho}_{(t)} / \omega_{l}\right) \sin \left(\omega_{l} t\right)+\rho_{0} \cos \left(\omega_{l} t\right)$,

$Z(t)=\left(\dot{Z}_{r} / \sqrt{2} \omega_{l}\right) \sinh \left(\sqrt{2} \omega_{l} t\right)$

$$
+Z_{t} \cdot \cosh \left(\sqrt{2} \omega_{l} t\right)
$$

where $\rho_{0}, \dot{\rho}_{0}$, and $Z_{c}, \dot{Z}_{c}$ are the initial conditions at $t=0$ on the top-cap electrode. $\dot{\rho}_{0}$ and $\dot{Z}_{t}$ are given by

$$
\begin{aligned}
& \dot{\rho}_{0}=\left[2 q\left(\phi_{T}-\phi_{c}\right) / m\right]^{1 / 2} \sin \epsilon, \\
& \dot{Z}_{c}=\left[2 q\left(\phi_{T}-\phi_{c}\right) / m\right]^{1 / 2} \cos \epsilon,
\end{aligned}
$$

where $q \phi_{T}$ is the kinetic energy of the charged particle before entering the analyzer and $\epsilon$ is the angle between the initial particle direction and the $Z$ axis of the analyzer.

The following expression can be derived from Eqs. (8) and (9) after eliminating $t$ :

$$
\rho(Z)=\sqrt{2} \alpha Z_{r} \cdot \tan \epsilon \sin \theta+\rho_{0} \cos \theta,
$$

where $\theta$ and $\alpha$ are functions of the ratios of physical quantities and are given by

$$
\theta=\frac{1}{\sqrt{2}} \log \left(\frac{Z / Z_{c}-\left[\left(Z / Z_{c}\right)^{2}-\left(1-\alpha^{2}\right)\right]^{1 / 2}}{1-\alpha}\right)
$$

and

$$
\alpha=\left(\frac{\phi_{T}-\phi_{C}}{\phi_{B}-\phi_{C}} \frac{\rho_{c}{ }^{2}+2 Z_{0}{ }^{2}}{2 Z_{c}{ }^{2}}\right)^{1 / 2} \cos \epsilon .
$$

Thus a choice of a particular $\theta$ or $\alpha$ does not dictate a particular physical dimension to be used. 


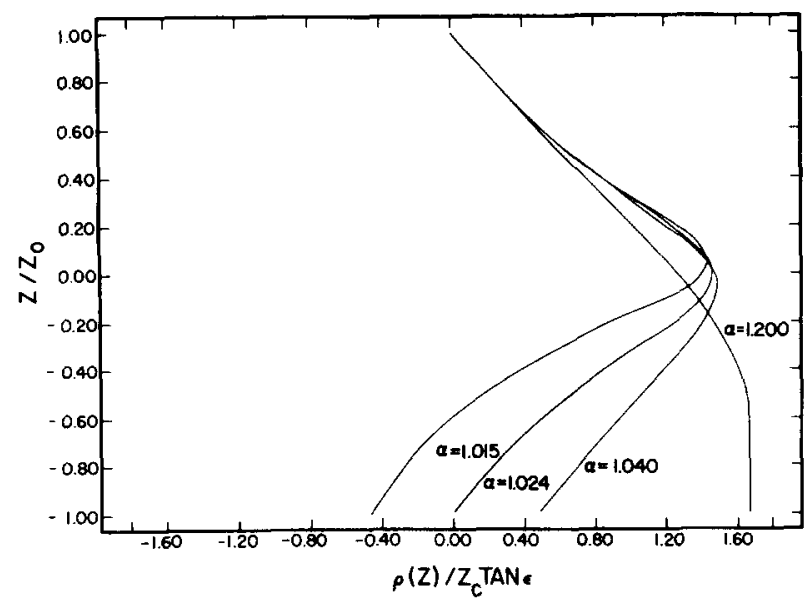

(a)

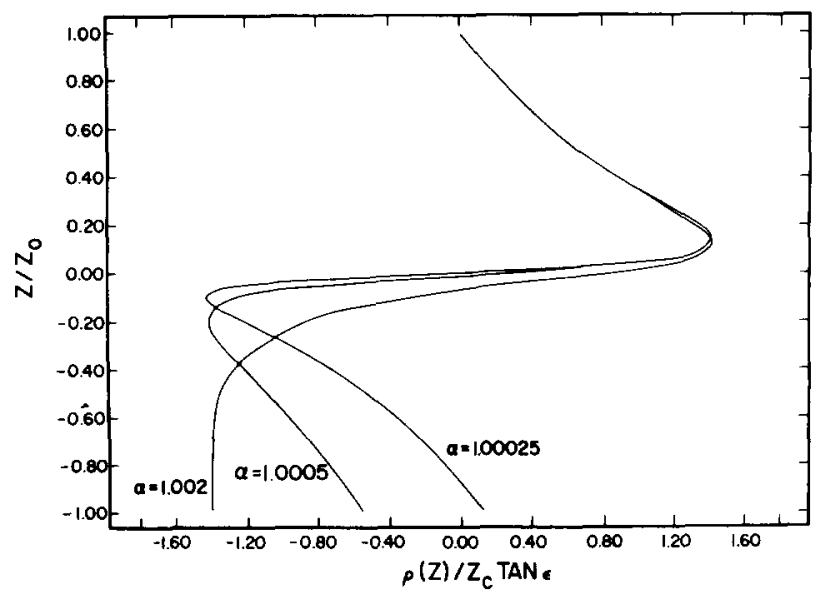

(b)

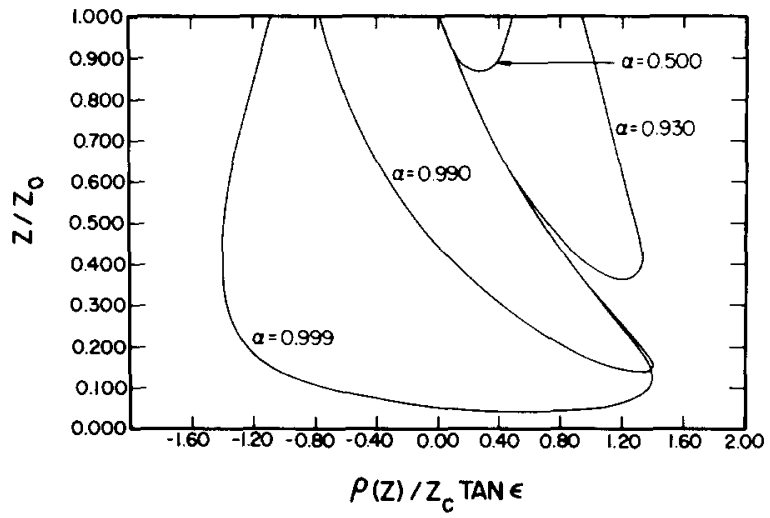

(c)

FIG. 2. (a) First-order trajectories for various $\alpha(\alpha>1)$ of a charged particle in the retarding mode when $\rho_{01}=0, \epsilon \neq 0$. (b) Second-order trajectories of a charged particle in the retarding mode when $\rho_{0}=0$ and $\epsilon \neq 0$ for various $\alpha(\alpha>1)$. (c) Charged particle trajectories in the retarding mode when $\rho_{0}=0$ and $\epsilon \neq 0$ and various $\alpha(\alpha<1)$.

Equation (12) can be written

$$
\frac{\rho(Z)}{2 Z_{c} \tan \epsilon}=\frac{1}{\sqrt{2}} \alpha \sin \theta+\frac{\rho_{0}}{2 Z_{c} \tan \epsilon} \cos \theta .
$$

There are four possible cases for combinations of $\rho_{0}$ and $\epsilon$.

Case a: $\rho_{0}=\epsilon=0$. The trajectory is a straight line along the $Z$ axis. This case corresponds to the conventional retarding potential method. The cutoff potential is the potential at $Z=0$ when the potential is equal to the kinetic energy of the particle.

Case $b: \rho_{0}=0, \epsilon \neq 0$. Particle trajectories of several $\alpha$ values are shown in Figs. 2(a) and 2(b). It is noted that the figure is a universal one because the figure has been plotted in terms of $\alpha$ and the ratios of $\rho_{\%}$ and $Z_{c} \cdot \tan \epsilon$, all of which are internal quantities as noted above.

As shown in the figure, the trajectories diverge from the origin in the upper half of the cavity and converge toward the bottom cap in the lower half. The trajectories are rapidly oscillating as $\alpha$ approaches unity and have nodal points depending upon the rapidity of the oscillation, which is a characteristic of wave motion. Therefore, there is an optical analog for a retarding mode. The trajectories are called first order when there is no other nodal point in the region $Z=Z_{0}$ and $-Z_{0}$, second order when there is one nodal point in this region, and so on for higher orders.

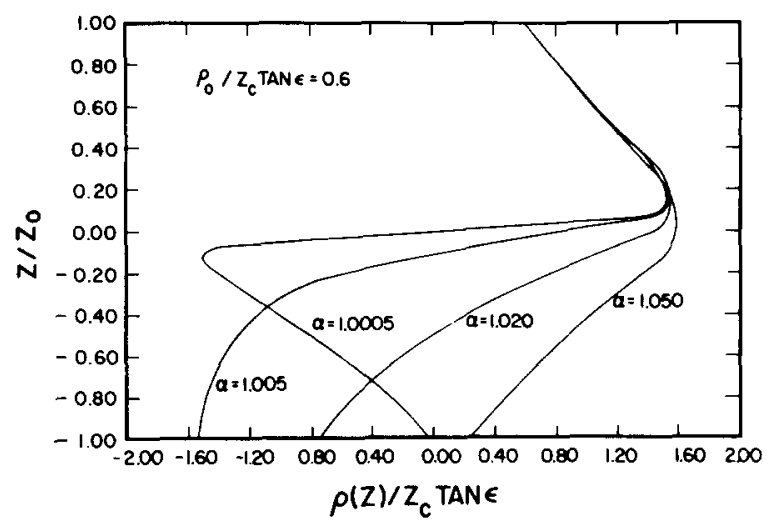

(a)

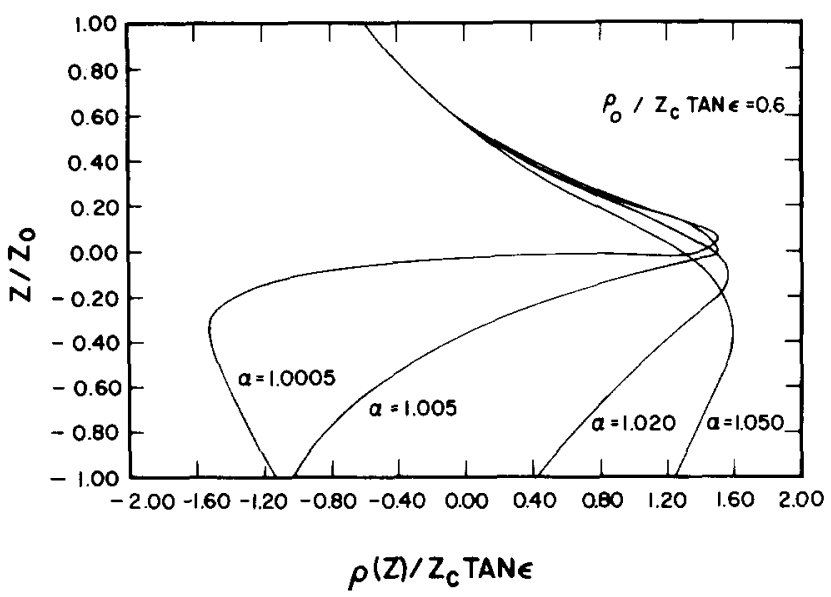

(b)

FIG. 3. (a) Trajectories for various $\alpha$ of a charged particle incident away from $\rho=0\left(\rho_{0} \neq 0, \epsilon \neq 0\right.$, phase advance). (b) Trajectories for various $\alpha$ of a charged particle incident toward $\rho=0\left(\rho_{0} \neq 0, \epsilon \neq 0\right.$, phase lag). 


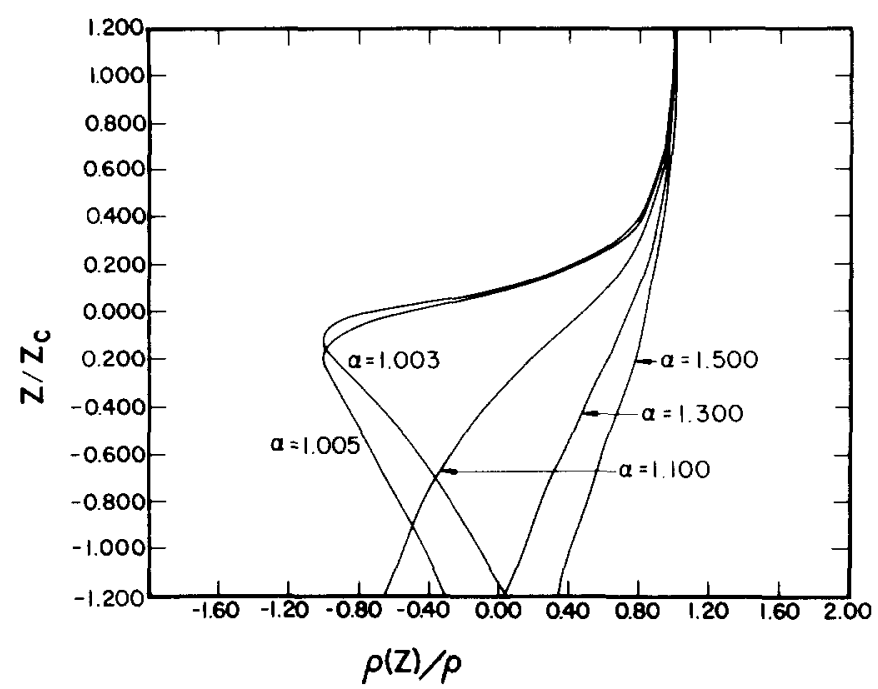

Fici. 4. Trajectories for various $\alpha$ of a charged particle when $\epsilon=0, \rho_{01} \neq 0$. It is noted that there is a phase advance by one-qualter $(\pi / 2)$ of a wavelength.

Figure 2(a) shows the first-order trajectories, which are relatively less sensitive to the variation of $\alpha$. Figure 2(b) shows the second-order trajectories, which have a strong dependence on $\alpha$. Note here that the end point at $Z=-Z_{10}$ moves rapidly across the end-cap. Higher order trajectories will have an even stronger dependence on $\alpha$, resulting in a more rapid movement of the end point of the trajectory across the end-cap. Figure 2(c) shows the particle trajectory when the energy is insufficient to overcome the potential barrier at $Z=0(\alpha<1)$. The trajectory turns back toward $Z=+Z_{0}$, resulting in a complete cutoff of the lower half of the cavity from the upper half.

Case $\because: \rho_{n} \neq 0, \epsilon \neq 0$. This case has generally the same trajectories as the previous case except that there are two different initial conditions. One is a diverging trajectory (away from $\rho=0$ ) as shown in Fig. 3(a), and the other is a converging trajectory (toward $\rho=0$ ) in the upper half of the cavity as shown in Fig. 3(b).

When the trajectories are described in terms of the wave motion, the diverging trajectory has a phase advance initially and the other (converging) a phase lag.

Case $d: \epsilon=0, \rho_{0} \neq 0$. Here the trajectories are shown in terms of $\rho / \rho_{0}$ since tan $\epsilon=0$. This case has a phase shift lag of one-quarter of a wavelength initially in the trajectory. A few trajectories are shown in Fig. 4 that are similar to those in well known electrostatic lenses. ${ }^{13-1.5}$

\section{Accelerating mode}

In the accelerating mode, the coefficient $\omega_{l}{ }^{2}$ in Eqs. (5) and (6) is negative, which implies that $\phi_{B}>\phi_{\text {. for }}$ $q<0$ or $\phi_{k}<\phi_{c}$. for $q>0$. The solution of this mode with the same initial condition as in the previous mode is given by

$$
\rho(Z)=\sqrt{2}\left(Z_{r} \cdot \tan \epsilon\right) \alpha^{\prime} \sinh \theta^{\prime}+\rho_{0} \cosh \theta^{\prime},
$$

where

$$
\begin{aligned}
& \theta^{\prime}=\frac{1}{\sqrt{2}} \\
& \times \tan ^{-1}\left(\frac{\alpha^{\prime}\left(Z / Z_{c}\right)-\left[1+\alpha^{\prime 2} \cdots\left(Z / Z_{r}\right)^{2}\right]^{122}}{\left(Z / Z_{\alpha}\right)+\alpha^{\prime}\left[1+\alpha^{\prime 2}-\left.\left(Z / Z_{1}\right)^{2}\right|^{122}\right.}\right)
\end{aligned}
$$

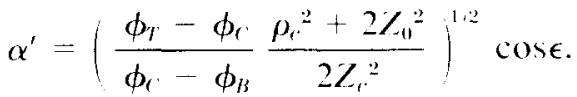

Similar to the retarding mode. there are four cases. but there is no optical analogy as in the retarding mode.

Case $a: \epsilon=0, \rho_{0}=0$. This case is a straight line trajectory along the $Z$ axis similar to the retarding case except that there is no cutoff potential.

Case $b: \epsilon \neq 0, \rho_{0}=0$. Trajectories of several $\alpha x^{\prime}$ are shown in Fig. 5. The trajectories converge to the origin in the upper half of the $Z$ axis and diverge at $-Z_{11}$ in the lower half. Also, note that as $\alpha^{\prime}$ hecomes zero the trajectory is approaching the $Z$ axis monotonically. regardless of the incidence angle $\epsilon$. i.e.. $\phi_{B}$ is very large compared to the kinetic energy of the charged particle.

Case $\left(: \epsilon \neq 0, \rho_{0} \neq 0\right.$. Equation (16) can be written ats

$$
\rho(Z) / \rho_{0}=\left(\sqrt{2} Z_{c} \tan \epsilon \rho_{0}\right) \alpha^{\prime} \sinh \theta^{\prime}+\cosh \theta^{\prime}
$$

The trajectories for a few $\alpha^{\prime}$ values are shown in Fig. 6. As shown in the figure, one trajectory (increasing $\rho)$ always diverges monotonically from the $Z$ axis in the $-Z$ plane. The other trajectory (decreasing $\rho$ ) crosses the $Z$ axis (in the $-Z$ plane) before diverging from the $Z$ axis.

Case $d: \epsilon=0, \rho_{0} \neq 0$. In this case, the trajectories are similar to the previous case (increasing $\rho$ ), in which the trajectory is diverging from the $Z$ axis monotonically.

The divergence of the trajectories appears to violate a general rule of electron optics. ${ }^{1 t i}$ That general rule has been derived from an approximate solution (i.e., particle trajectories are assumed to have a small radial distance from the axis), but the present result is an exact solution of the quadrupole field.

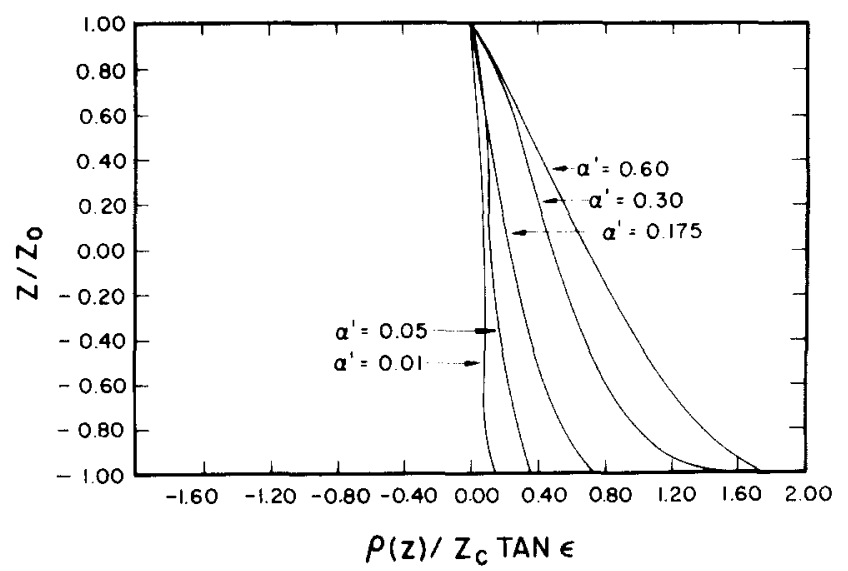

FIG. 5. Trajectories for various $\alpha$ of a charged particle in the accelerating mode when $\epsilon \neq 0, \rho_{11}=0$. 


\section{Energy resolution}

If a small (circular) aperture is located anywhere on the lower-cap electrode, the particles within a small energy range about a certain energy can be transmitted and collected selectively.

The energy resolution for the retarding mode is derived from Eq. (14), where the incident energy, $E$, of the charged particle can be expressed as a function of two independent variables, $\alpha$ and $\epsilon$,

$$
\frac{\Delta E}{E}=\frac{\Delta q\left(\phi_{r}-\phi_{r^{\prime}}\right)}{q\left(\phi_{T}-\phi_{r^{\prime}}\right)}=\frac{2 \Delta \alpha}{\alpha}+2 \tan \epsilon(\Delta \epsilon) .
$$

For the accelerating mode, the same equation applies with the replacement of $\alpha$ by $\alpha^{\prime}$. The first term in Eq. (20) is determined by the radii of the collecting aperture and the entrance aperture. The second term is determined by the field of view of the energy analyzer. $\Delta \alpha$ (or $\Delta \alpha^{\prime}$ ) is the difference between the two $\alpha$ values which determine the energy window through the collecting aperture. and $\Delta \epsilon$ is the angle of the field of view at the entrance slit. It should be noted that $\Delta E$ in Eq. (20) is the baseline energy difference, not the half-energy width.

In order to calculate the first term in the energy resolution [Eq. (20)], for an infinitesimal entrance aperture [the calculation for a finite size aperture (a beam) will be discussed later], the impact radius of the particle trajectory, $\rho_{I}$, is defined at $Z=-Z_{n}$. for both modes.

$$
\begin{array}{r}
\rho_{t}^{(\prime \prime)}(\alpha)=\sqrt{2} \alpha Z_{c} \cdot \tan \epsilon \sin \left[\frac{1}{\sqrt{2}} \log \left(\frac{\alpha+1}{\alpha-1}\right)\right] \\
+\rho_{0} \cos \left[\frac{1}{\sqrt{2}} \log \left(\frac{\alpha+1}{\alpha-1}\right)\right]
\end{array}
$$

for the retarding mode and

$$
\begin{aligned}
\rho_{l}^{(\prime \prime \prime}\left(\alpha^{\prime}\right)= & \sqrt{2} \alpha^{\prime} Z_{,} \tan \epsilon \sinh \left[\frac{1}{\sqrt{2}} \tan ^{-1}\left(\frac{2 \alpha^{\prime}}{1-\alpha^{\prime 2}}\right)\right] \\
& +\rho_{0} \cosh \left[\frac{1}{\sqrt{2}} \tan ^{-1}\left(\frac{2 \alpha^{\prime}}{1-\alpha^{\prime 2}}\right)\right]
\end{aligned}
$$

for the accelerating mode. A plot of the ratios $\rho_{I} / 2 Z_{\text {r }}$ $\tan \epsilon$ (or $\left.\rho_{l} / \rho_{0}\right)$ vs $\alpha\left(\right.$ or $\alpha^{\prime}$ ) is termed an "impact radius plot" for convenience.

When the boundaries of the collecting aperture are $\rho_{1}$ and $\rho_{2} . \Delta \alpha$ is given by

$$
\Delta \alpha=\left|\alpha_{1}-\alpha_{2}\right|,
$$

where $\alpha_{1}$ and $\alpha_{2}$ satisfy the following relationship:

$$
\begin{aligned}
& \rho_{1}=\rho_{l}\left(\alpha_{1}\right), \\
& \rho_{2}=\rho_{l}\left(\alpha_{2}\right) .
\end{aligned}
$$

It is convenient to find the $\alpha_{1}$ and $\alpha_{2}$ value from the impact radius plot. As the radius of the collecting aperture and the angle of the field of view are increased, the energy resolution becomes poorer.

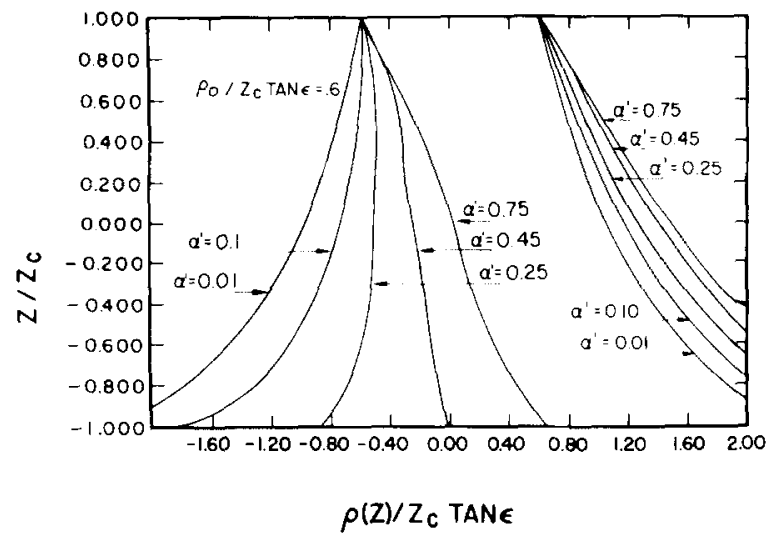

FIg. 6. Two kinds of trajectories for various $\alpha$ in the accelerating mode when $\rho_{0} \neq 0, \epsilon \neq 0$. Trajectories in left of figure (converging) sweep across the center axis of the end-cap. while the trajectories on the right side (diverging) do not.

\section{Returding mode}

Case $a: \epsilon=0, \rho_{0}=0$. This case has zero impact radius regardless of the kinetic energy and is of no interest to the present work.

Case $b: \epsilon \neq 0, \rho_{0}=0$. The impact radius plot is shown in Fig. $7\left(\rho_{l} / 2 Z_{\text {, }}\right.$ tan $\epsilon$ vs $\alpha-1$, where $2 Z_{c}$, tan $\epsilon$ is the impact radius at infinite energy). As shown in the figure the rapid oscillation in the impact radius occurs very close to the cutoff energy, which corresponds to the case $\alpha=1$. at which the energy is given by

$$
E=q\left(\phi_{T}-\phi_{c^{\prime}}\right)=\frac{2 q\left(\phi_{B}-\phi_{c}\right) Z_{0}^{2}}{\rho_{c}{ }^{2}+2 Z_{1}{ }^{2}}-\frac{1}{\cos ^{2} \epsilon} .
$$

The higher order oscillation is so rapid that the most important energy selection takes place at the rather broad energy window. A, where the particle is first focused at the collecting aperture (first order).

Case $c: \epsilon \neq 0, \rho_{0} \neq 0$. Figure 8 shows the impact radius plot $\left(\rho_{l} / 2 Z_{r}\right.$. tan $\epsilon$ vs $\left.\alpha-1\right)$ for $\rho_{0} / Z_{r} \cdot \tan \epsilon=0.6$. As noticed in the figure, there are two energy windows for each order compared to one window for case $b$. The energy resolution for phase lag is improved relative to the

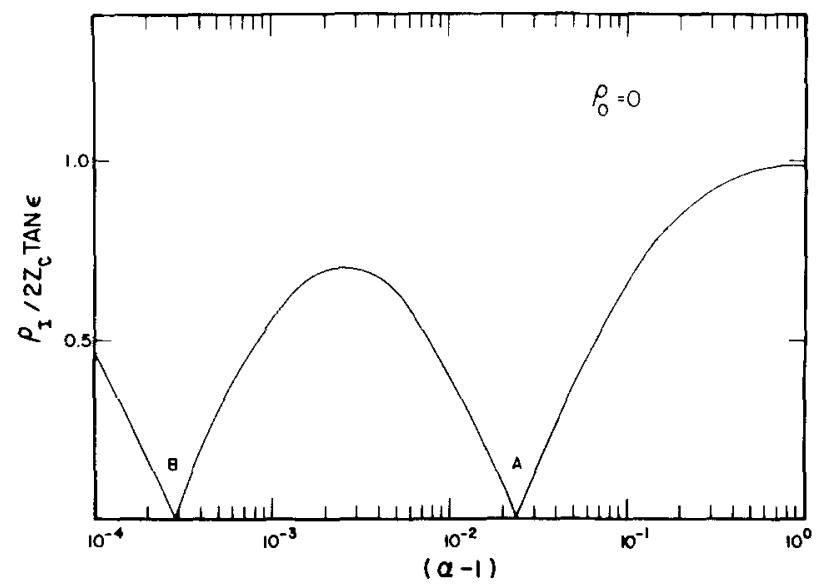

FIG. 7. Impact radius plot in the retarding mode. A indicates the first-order energy window and $B$ the second-order energy window when the collecting aperture is located at the center of the lower end-cap. 


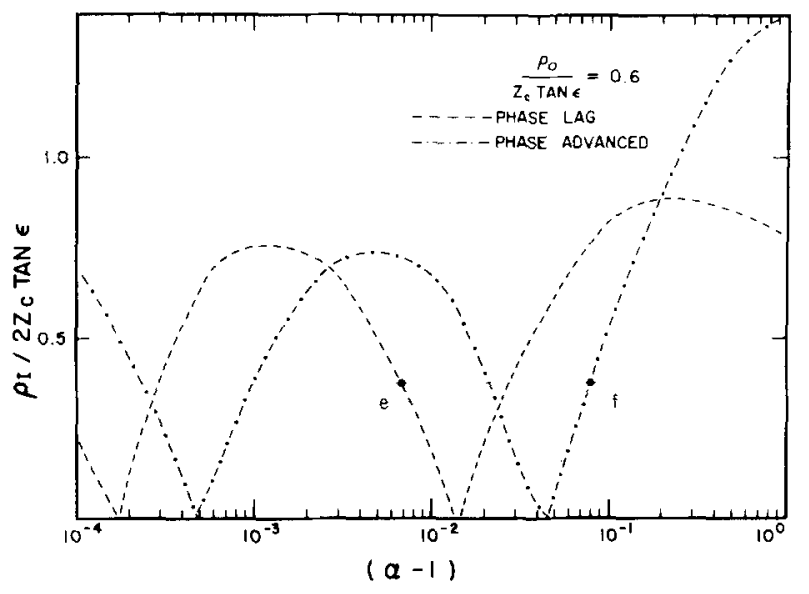

Fic;. 8 . Impact radius plot $\left(\rho_{i} / 2 Z_{\text {, tane }}\right.$ vs $\left.\alpha-1\right)$ for the retarding mode when $\epsilon \neq 0 . \rho_{0} \neq 0$. Here $\mu_{0} / Z_{r}$ tan $\varepsilon=0.6$. See text for discussion of phatse latg and phase advance.

case $b$, while for phase advance the resolution becomes worse.

Case $d: \epsilon=0, \rho_{0} \neq 0$. This is the limiting case for case $c$ above. The impact radius plot is shown in Fig. 9. It should be mentioned that there is another energy window, $A_{0}$, prior to the first-order energy window, A. However, the energy resolution is poorer when compared to case $b$. There is a minimum effect to the energy resolution by the field of view because $\tan \epsilon \equiv 0$ in Eq. (20).

\section{Accelerating mode}

Case $a: \epsilon=0, \rho_{11}=0$. The impact radius is zero and no energy analysis can be done in this case.

Case $b: \epsilon \neq 0, \rho_{0}=0$. The impact radius plot is shown in Fig. 10 and the impact radius contains the integrated transmission unless the collecting aperture is located off-axis because of the monotonic decrease of the impact radius as $\alpha^{\prime}$ decreases.

Case $c: \epsilon \neq 0, \rho_{0} \neq 0$. Again as for the retarding mode $\left(\epsilon \neq 0, \rho_{0} \neq 0\right)$, there are two impact radii depending upon the convergence or divergence of the trajectory. Figure 10 shows the converging trajectory only when $\rho_{10} / Z_{r}$. tan $\epsilon=0.6$. This case could be an interesting one because of a wider range of the impact radius.

Case $d: \epsilon=0, \rho_{0} \neq 0$. This impact radius increases monotonically from $\rho_{i}=\rho_{0}$ as $\alpha^{\prime}$ decreases.

Finally, the energy resolution [first term of Eq. (20)] is considered for an entrance aperture of a finite size $\left(\right.$ radius $\left.=\Delta \rho_{0}\right)$.

Using Eq. (21), two impact radius plots are calculated for the retarding mode when the initial position of the particle injection, $\rho_{0}$, is replaced by $\rho_{0} \pm \Delta \rho_{0}$. The two impact radius plots are shifted away from the case $\Delta \rho_{0}=0$ (a point aperture) on both sides. The difference between two extreme $\alpha$ values gives $\Delta \alpha$ for the energy resolution. For example, when the ratio of the radius of the entrance aperture at the center of the upper endcap to $Z_{\text {. }}$ tan $\epsilon$ is 0.6 , then the two impact radius plots are as shown in Fig. 8. The two extreme values of $\epsilon$ are

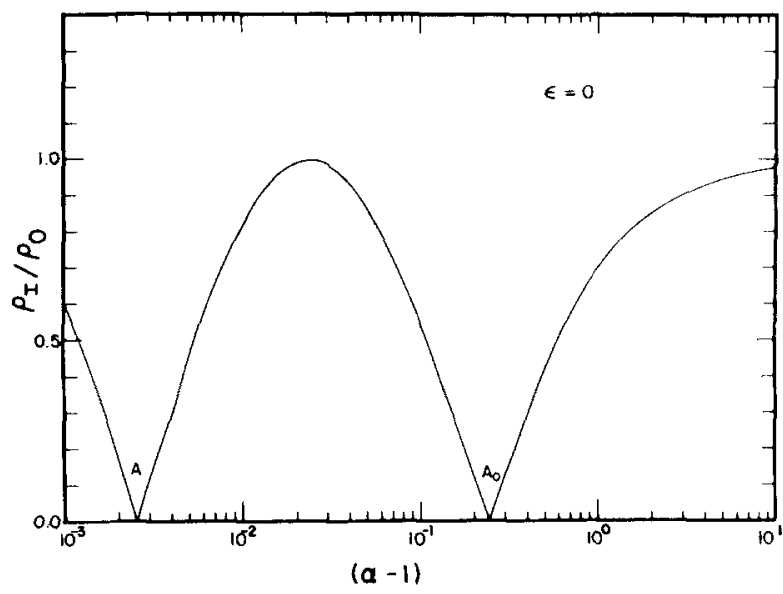

Fig. 9. Impade radius plot when $\epsilon=0$ and $p_{10} \neq 0 . A_{1}$ is the winkow for a phase lag of $\pi / 2$. A is the first-order window.

at points $e^{\prime}$ and $f$ for the collecting aperture located at the center of the lower-end-cap. Likewise, $\Delta \alpha^{\prime}$ for the accelerating mode can be found by using Fq. (22).

\section{EXPERIMENT}

The theory of the HARP with an electrostatic field has been developed in the previous section, and the experimental verification of the theory will be given in this section. A monoenergetic and directional electron beam wats used to inject electrons into the HARP. Both angular and energy analysis were done.

The experimental work has been accomplished with an existing apparatus that had previously been used for studying electron impact cross sections of gases. The HARP used in the present work was one which had been used in studying the energetic plasma surrounding the earth.

\section{A. Description of the apparatus}

A schematic diagram of the apparatus is shown in Fig. 11. The vacuum chamber is a cylindrical shape and a Vac-Ion pump (pumping speed — 500 liters/sec) pro-

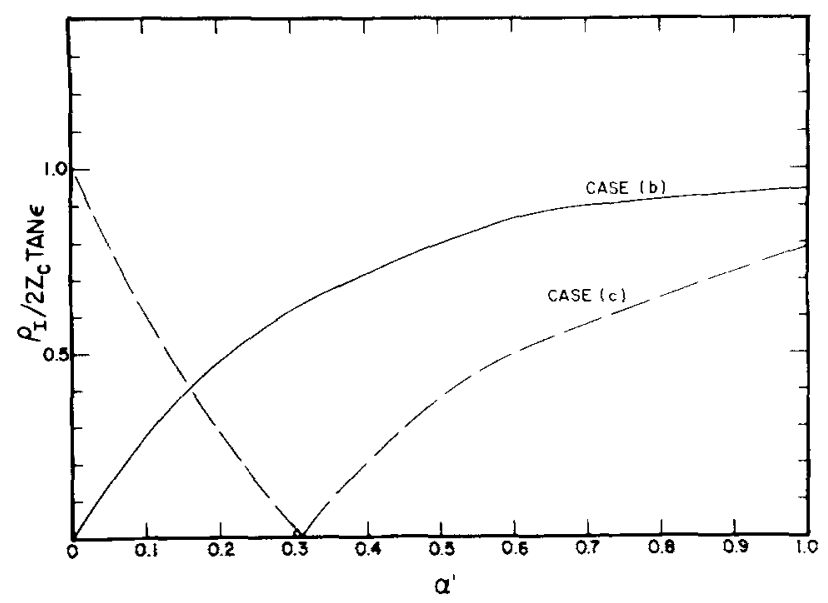

FIG. 10. Impact radius plot for the accelerating mode when $\epsilon \neq 0$, $\rho_{13}=0$ (case b) and $\epsilon \neq 0, \rho_{9} \neq 0$ (case $\left.c\right)$. 

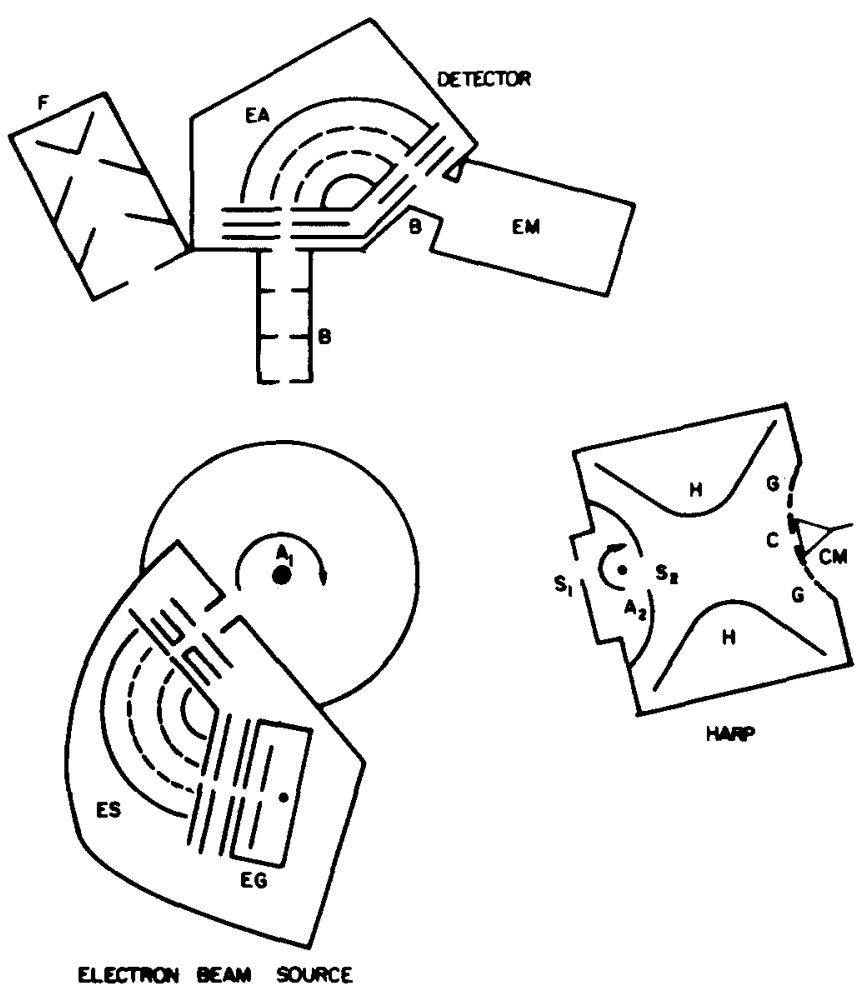

FIG. 11. Schematic diagram of apparatus. EG-Electron gun; ES$127^{\circ}$ electron energy selector; $A_{1}$ - rotational axis of electron beam source; $B$-baffle; EA-127 i energy analyzer; EM-electron multiplier; F-Faraday cup; $S_{1}, S_{2}$-entrance slits; $A_{2}$-rotational axis of HARP: $\mathrm{H}$-hyperbolic electrode; $\mathrm{C}$-exit aperture; $\mathrm{G}$-grid; CM-Channeltron multiplier.

vides an oil-free vacuum of $10^{-8}$ Torr. The apparatus consists of three subsystems: a rotatable electron-beam source and two energy analyzers (a fixed electron detector and a rotatable and horizontally movable HARP). Two sets of Helmholtz coils are used to compensate the residual magnetic field from the vacuum pump magnets and the earth's magnetic field down to $0.05 \mathrm{G}$ in all directions.

\section{Electron-beam source}

The electron-beam source is comprised of an electron gun and a $127^{\circ}$ electrostatic cylindrical energy selector. The resultant half-width of the energy-selected electron beam is $0.06 \mathrm{eV}$ independent of the energy. There are two electron-beam deflectors (horizontal and vertical) which are used to direct the electron beam in the precise direction desired. The highly directional electron beam has an angular half-width of about $2^{\circ}$ and produces a beam current of $10^{-10}-10^{-9} \mathrm{~A}$. A more detailed description can be found elsewhere. ${ }^{17}$

\section{Fixed electron detector}

This electron detector is a $127^{\circ}$ electron analyzer, which is the same device as was used in the electronbeam source. This energy analyzer along with a Faraday cup on the chamber wall is used to check the electron beam (energy spread and directionality) in the experiment.

\section{HARP}

The actual geometry of the HARP used in this experiment is shown in Fig. 12, and the dimensions of the HARP are given in Table I. The HARP central electrode is made of gold-plated aluminum and is surrounded on the outside by Co-Netic for magnetic shielding. The end-caps are formed from Co-Netic. This reduces the internal magnetic field to less than $0.01 \mathrm{G}$. Behind the exit aperture, the selected electrons are accelerated by $150 \mathrm{~V}$ into a Channeltron multiplier which serves as the detector. The present experimental setup is a special case of the various combinations between the entrance and exit aperture geometries, i.e., the entrance aperture with $\epsilon=15^{\circ}\left(\Delta \epsilon= \pm 4^{\circ}\right)$ and $\rho_{0}=0$ and the exit aperture on axis (the radius of the exit aperture $=0.35 \mathrm{~cm}$.) The field of view $\left(\Delta \epsilon= \pm 4^{\circ}\right)$ of the analyzer is determined by the width of the two slits, $S_{1}$ and $S_{2}$, shown in Fig. 11.

The HARP can be rotated and translated (horizontally and vertically) relative to the direction of the electron beam. The rotational axis of the analyzer is located at the intersection of the two extreme rays between the two slits of the entrance aperture.

There is an electron trap electrode in concentric rings about the detector behind the exit end-cap. The trap electrode is biased $+50 \mathrm{~V}$ relative to the exit end-cap electrode. The trap is effective for electrons with energy above the selected energy in the detector and secondary electrons produced by the energetic incident electrons.
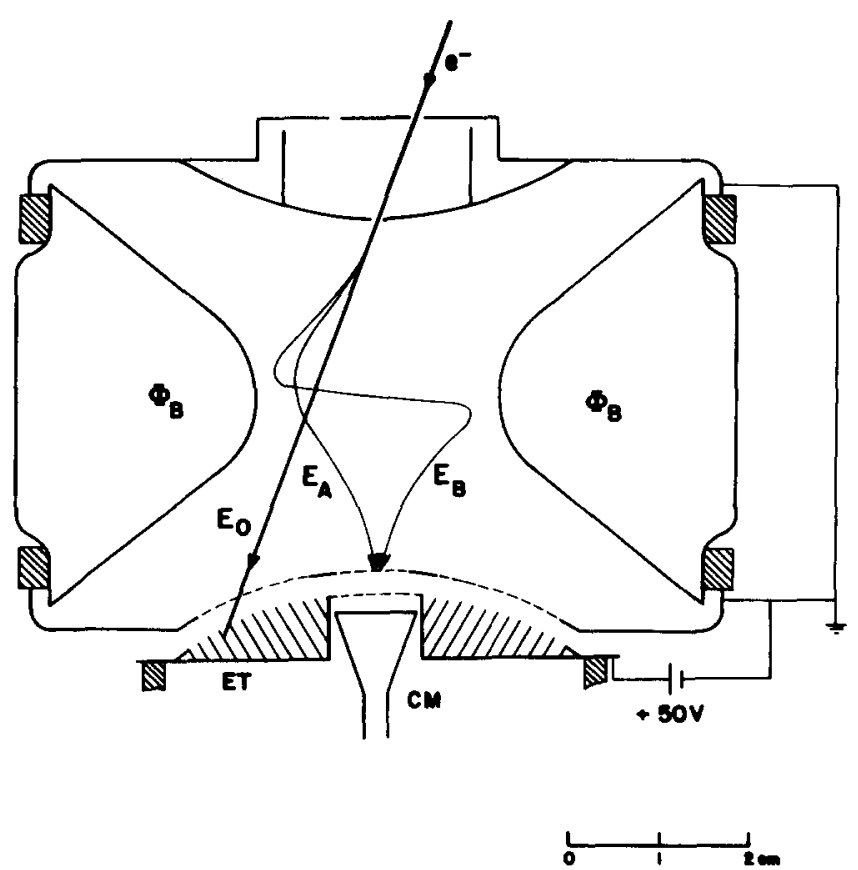

FIG. 12. Schematic of the HARP. $\phi_{B}$ is the selection potential on the hyperbolic electrode. $E_{0}$ is an infinite energy with a straight line trajectory. $E_{A}$ is a first-order and $E_{B}$ is second-order energy for a given $\phi_{B}$ when the beam is focused at the exit. Here $\mathrm{E}_{B}<\mathrm{E}_{A}$. ET is an electron trap which is biased $+50 \mathrm{~V}$ relative to the end-cap electrode. 
TABLE 1. Dimensions of HARP

\begin{tabular}{rlrl}
\hline$\rho_{r}=1.27 \mathrm{~cm}$ & $S_{1}$ & $=0.191 \mathrm{~cm}$ (width) \\
$Z_{01}=1.91 \mathrm{~cm}$ & $S_{2}$ & $=0.046 \mathrm{~cm}$ (radius) \\
$\epsilon$ & $=15^{\circ}$ & $C$ & $=0.35 \mathrm{~cm}$ (radius) \\
$\Delta E$ & $= \pm 4^{\circ}$ & $\phi_{b} / E$ & $=1.076 \mathrm{at}$ the center of energy window
\end{tabular}

\section{B. Procedure}

The electron-beam source is stabilized over a 2-h warmup period. The potential $\phi_{B}$ on the hyperbolic electrode is swept by a function generator for a given electron beam energy from the electron beam source (setting $\left.\phi_{i^{\prime}}=0\right)$.

The electron current of the selected energy is collected on the Channeltron electron multiplier. The output pulses from the multiplier are fed through a pulse amplifier to a counter. The output counts are recorded on an $\mathrm{X}-\mathrm{Y}$ recorder through a $\mathrm{D} / \mathrm{A}$ converter.

\section{Results}

\section{Retarding mode}

The energy response of the analyzer has been measured for a fixed incident angle $\left(\epsilon=15^{\circ}\right)$ for various incident energies $(1.3,3,4,5,10,20,50,100,200,300$, 400 , and $500 \mathrm{eV}$ ) by sweeping the potential on the hyperbolic electrode. Figure 13 shows the typical response at three incident energies. First-order and sharp secondorder windows are clearly evident at $\mathrm{A}$ and $\mathrm{B}$, respectively, in Fig. 13. The high-order passbands do not appear in the experimental data because the count rate is below detectability owing to the extremely narrow energy windows.

The theoretical energy resolution given by Eq. (20) at a fixed angle $\left[(\Delta E / E)_{\Delta \epsilon=0}=2 \Delta \alpha / \alpha\right]$ is $7.8 \%$ when the finite width of the entrance slit is considered.The mean experimental energy resolution is $(7.5 \pm 0.5) \%$ for the various incident energies. The agreement between the theoretical and experimental values is relatively good.

A typical angular response of the analyzer at an incident energy of $20 \mathrm{eV}$ is shown in Fig. 14. It is noted that at smaller incident angles the energy window is broader. Also, the center of the energy window is shifted toward lower $\phi_{l s}$ as the incident angle is increased. The reason for this is that for a fixed $\alpha_{1}$ and $\alpha_{2}$ (also $\Delta \alpha$ ), which are determined by the geometry of the exit aperture, there is a functional relationship between $\phi_{B}$ and $\epsilon$ [viz., Eq. (14)]. The fractional energy width broadening due to the field of view of the analyzer, given by $2 \tan \epsilon(\Delta \epsilon)$ in Eq. (20), should be added to $2 \Delta \alpha / \alpha$ to obtain the total energy resolution. A theoretical value for $2 \tan \epsilon(\Delta \epsilon)$ is $7.5 \%$ when $\epsilon=15^{\circ}$ and $\Delta \epsilon=8^{\circ}$. Thus the total theoretical value of $\Delta E / E$ is $15.3 \%$.

A typical response of the analyzer to an isotropic electron source of $20 \mathrm{eV}$ energy can be obtained simply by adding the response at all angles in the field of view as shown in Fig. 15. The result is roughly triangular shaped. Here $\Delta E /\left.E\right|_{\text {baseline }}=15.5 \%$ and $\Delta E /\left.E\right|_{\text {half-width }}$ $=9.0 \%$. Consideration of the response at other energies

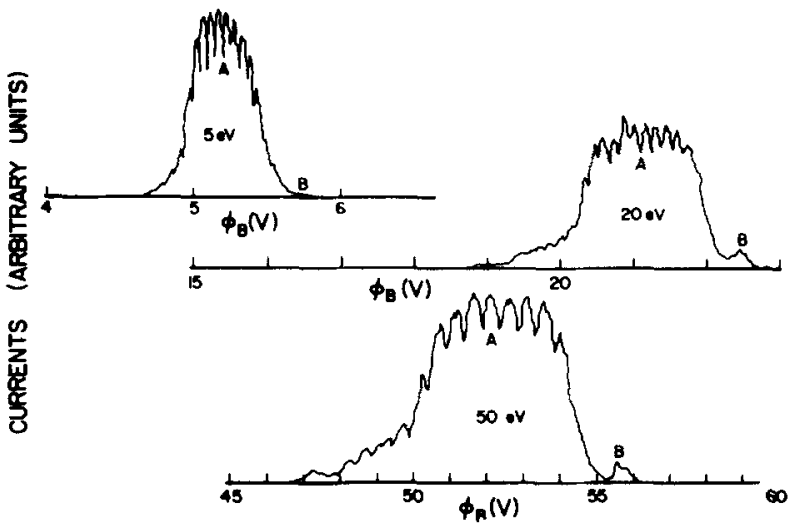

FIG. 13. Typical response of the HARP at three incident energies (5. 20 , and $50 \mathrm{eV}$ ). $A$ is the hroad first-order encrgy window. and $B$ is the sharp secondzorder. The sawtooth nature of the data is due to the fact that the focused beam is dispersed in energy much less thith the grid spacing in front of the multiplier.

gives $\Delta E /\left.E\right|_{\text {baseline }}=15.5 \pm 0.5 \%$, which compares well with the theoretical resolution above.

\section{Accelerating mode}

The present geometry of the HARP (slit arrangement and exit aperture) is inappropriate to test the behavior of the electron beam in the analyzer in this mode. However, that the electron beam is focused and collected at the detector as $\phi_{I}$ increases is demonstrated by using positive voltages on the hyperbolic electrode and deflecting electrons. The result is shown in Fig. 16.

As shown in Fig. 10 (case b), the impact radius decreases monotonically as the applied potential $\phi_{l}$ increases. A lower limit of the impact radius is zero when the potential $\phi_{B}$ is infinite. Thus, at large $\phi_{B}$ values the integrated signal of all of the particles is collected and the count is approximately constant. The present geometry of the collecting aperture (radius $0.35 \mathrm{~cm}$ at the center of the lower end-cap electrode) implies that $\rho_{I}$ must be $0.35 \mathrm{~cm}$ or less to collect the electrons at it given energy. At $\rho_{l}=0.35 \mathrm{~cm}$, the detector starts

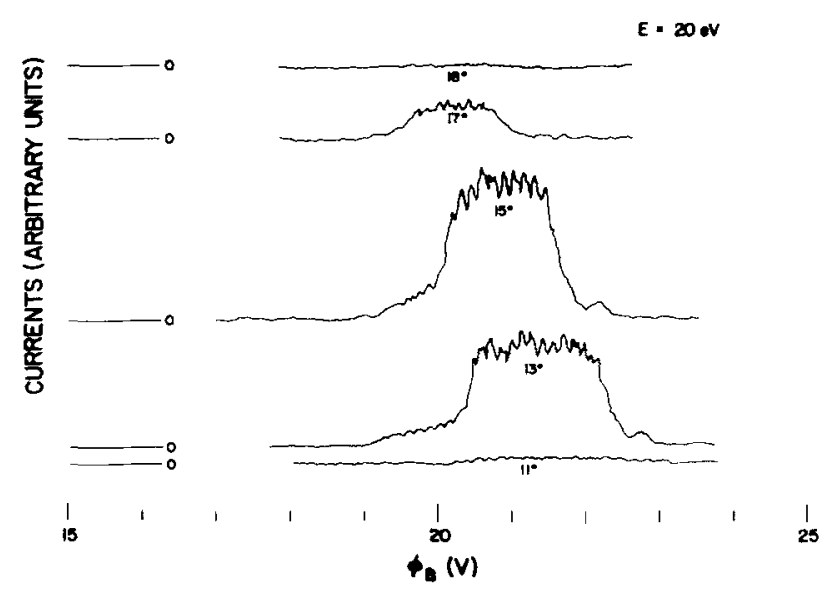

Fig, 14. Angular response of the HARP at an incident energy of $20 \mathrm{eV}$. At smaller incident angles, the energy window is broader. The center of the energy window shifts toward lower $\phi_{S}$ as the inciden angle is increased. 


\section{$E=20 \mathrm{eV}$}

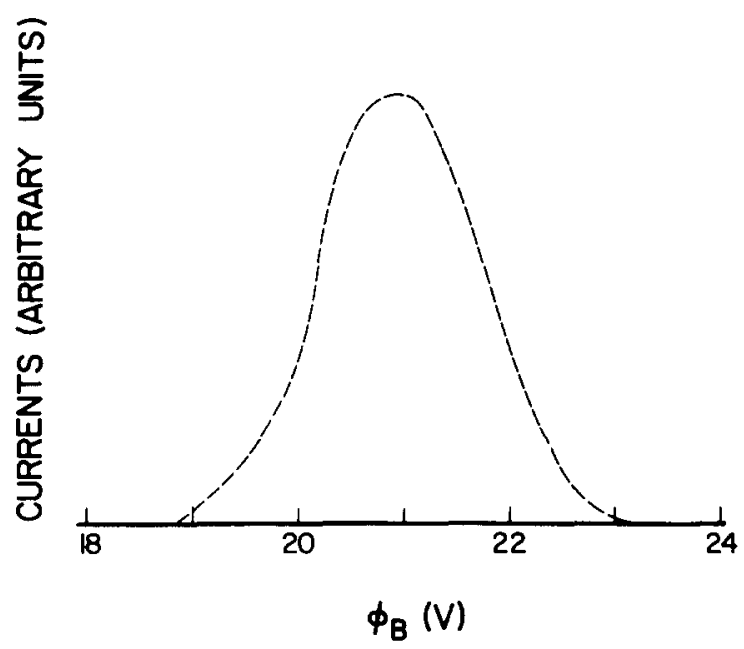

Fici. 15. Response of the HARP to an isotropic electron heam of $20 \mathrm{eV}$ energy.

collecting electrons. This corresponds to an $\alpha$ of approximately 0.15 , which gives the ratio of $\phi_{b}$ to this incident energy of the electron beam of approximately 30 . Since there is a large ratio of $\phi_{B}$ to the incident energy of the electron beam, the HARP may be useful to calibrate the absolute energy scale of an incident electron beam because the effect of the contact potential on the absolute energy scale is reduced by a factor of 30 .

\section{APPLICATIONS}

The principal application of the HARP to date has been as a rocket-borne detector of low-energy electrons in the ionosphere of the earth. Measurement of the energy distribution of ionospheric electrons for energies generally less than $100 \mathrm{~V}$ requires that several detection problems be considered which may not necessarily be present in laboratory uses of the instrument. The choice of the appropriate sensor is the primary problem. When the choice of the sensor is made, the operational mode must be maximized for detecting low-energy electrons. Then the problem of the charge state of the spacecraft must be considered, since that charge may forbid entry of low-energy electrons to the sensor. Finally, all electrons not of ionospheric origin must be determined and minimized.

\section{A. Sensor}

The use of the HARP for measuring low-energy electrons is based on three important considerations. (1) As noted in Sec. II, the HARP uses a selection potential on the central electrode that is near the energy of the collected electrons, whereas, in a conventional spherical or cylindrical analyzer normally used for space flight, only a small fraction of the electron energy is used for deflection. Thus, at low energies the HARP is significantly less sensitive to small potential irregularities on the electrodes. Also, for the same particle energy resolu- tions, the HARP requires a less precise selection potential than other analyzers. (2) All electrons being selected spend about $10 \%$ of their trajectory near an electrode wall in the HARP. This will result in a less significant influence on the trajectories from contact potential problems, whereas, in the spherical and cylindrical analyzers, electrons follow a wall throughout the time spent in the analyzer. At low energies, surface potential fluctuations can cause serious trajectory deformation near walls unless great care is taken. The nonproximity of walls to the trajectories in the HARP results in a distinct advantage in the measurement of the energy spectrum below a few volts. (3) For any group of analyzers, all having the same energy resolution, $\Delta E_{1: 2} / E$, the important quantity to consider is the product of the acceptance solid angle, $\Omega$, and the source area, $A$. For the HARP, as generally used, this is $8 \times 10^{-+} \mathrm{cm}^{2} \mathrm{sr}^{\circ}$ at $\Delta E_{1 / 2} / E$ $=9 \%$. This results in statistically significant counts and sufficient resolution to resolve any structure in the energy distribution. A disadvantage of the HARP is that a practical upper limit to the sampled electron energy exists because of the near-unity ratio of sampled to applied voltage. Kilovolt and higher energies are not sampled.

\section{B. Operational mode}

The retarding mode of operation is used since higher electron energies may be sampled than in the accelerating mode. Electrons enter the instrument at an angle of $\epsilon$ $=15^{\circ} \pm 4^{\circ}$ and on axis $\rho_{0}=0$. This is case $\mathrm{b}$ discussed in Sec. II. The case $c$ mode $\left(\rho_{0} \neq 0, \epsilon \neq 0\right)$ hats also been used, principally in an effort to study the effects of baffling scattered sunlight.

It should be recalled that both the accelerating and retarding modes operate simultaneously. If electrons are being deflected in the retarding mode, then positive ions are being deflected in the accelerating mode. Thus, steps must be taken to eliminate the undesired particles (ions in this application). When this is not done, the count rates at high body potentials will reflect the accelerating mode particles (ions) rather than the retarded particles (electrons) since the accelerated particles are $>10^{\circ}$ times more abundant than the retarded particles. The ions can be reflected from the lower end-cap to the central elec-

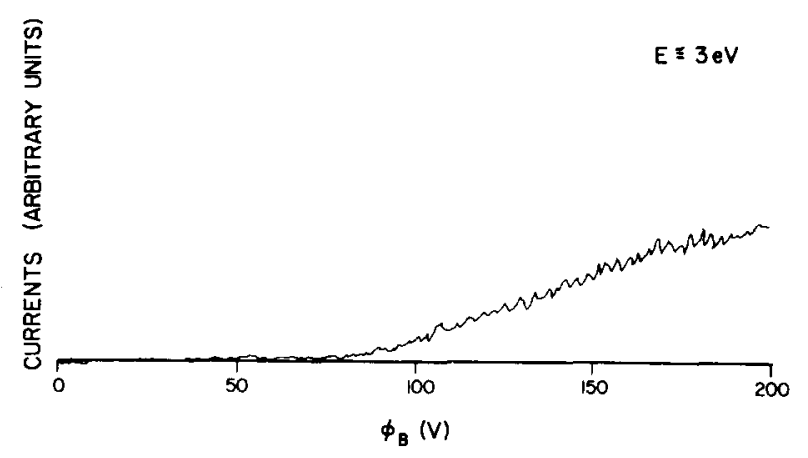

FIG. 16. Response of the HARP to an incident beam energy of $3 \mathrm{eV}$ in the accelerating mode. The signal begins to be collected at approximately $\phi_{l s}=90 \mathrm{~V}$. 
trode and produce secondary electrons which accelerate to the end-cap and contribute significantly to the count rate.

\section{Spacecraft charging}

In most circumstances spacecraft have a potential of -1 to $-3 \mathrm{~V}$. On some occasions, particularly with satellites, the potential can be considerable, for example, $20 \mathrm{~V}$ on OGO-F ${ }^{18}$ and kilovolts on ATS-5. ${ }^{19}$ The absolute energy basis of the sampled electrons depends upon the knowledge of the spacecraft charge state. A method has been designed and tested to measure this charge state. ${ }^{20}$ This method is based upon an intrinsic feature of the sensor in which the instrument reference potential is servoed to compensate the spacecraft potential. Upon finding the spacecraft potential the reference potential is held fixed for the energy sweep.

\section{Local electrons}

Electrons entering the sensor are called "local" if their origin, by production or reflection, is the instrument slits or spacecraft. The field of view of the sensor will see these local electrons in certain conditions of sensor-spacecraft-sun geometries, and spacecraft-magnetic field geometries. Any geometry is undesirable which results in a photoelectron from the spacecraft surface spiraling about the magnetic field into the sensor. Photoelectrons from the instrument slit are undesirable as well. The instrument is blinded to ambient electrons if any part of the spacecraft occupies space that the electron would occupy in its trajectory about the magnetic field into the sensor.

To overcome these problems the instrument is mounted on the forward end of the rocket payload with the entrance cone forward. Local electrons created at surfaces will not have the necessary trajectories to enter the HARP, nor will the HARP be blinded to ambient electrons. Furthermore, the rocket is launched when it is well assured that the sun-sensor angle will be about $90^{\circ}$ (dusk or dawn), which minimizes electrons created on the entrance slit. As noted in the previous section, electrons scattered inside the instrument are trapped in a Faraday cup arrangement after the bottom end-cap electrode.

\section{SOME EXPERIMENTAL RESULTS}

The HARP has been flown on several occasions and has successfully measured the electron distribution from 0.5 to $500 \mathrm{eV}$ in the earth's ionosphere, both during the day and in the aurora at night. The conversion from detector count rate, $C$, to flux is achieved by evaluating the integral ${ }^{21}$

$$
C=\frac{1}{\tau} \int_{\tau_{0}}^{\tau_{0}+\tau} d t \int_{A} \mathbf{r} \cdot d \mathbf{a} \int_{\Omega} d \Omega \int_{0}^{\infty} \epsilon(E) F(E, t) d E,
$$

where $E=$ particle energy, $\tau=$ sampling period, $\mathbf{r}=$ unit vector in direction $\Omega$, da $=$ surface area element

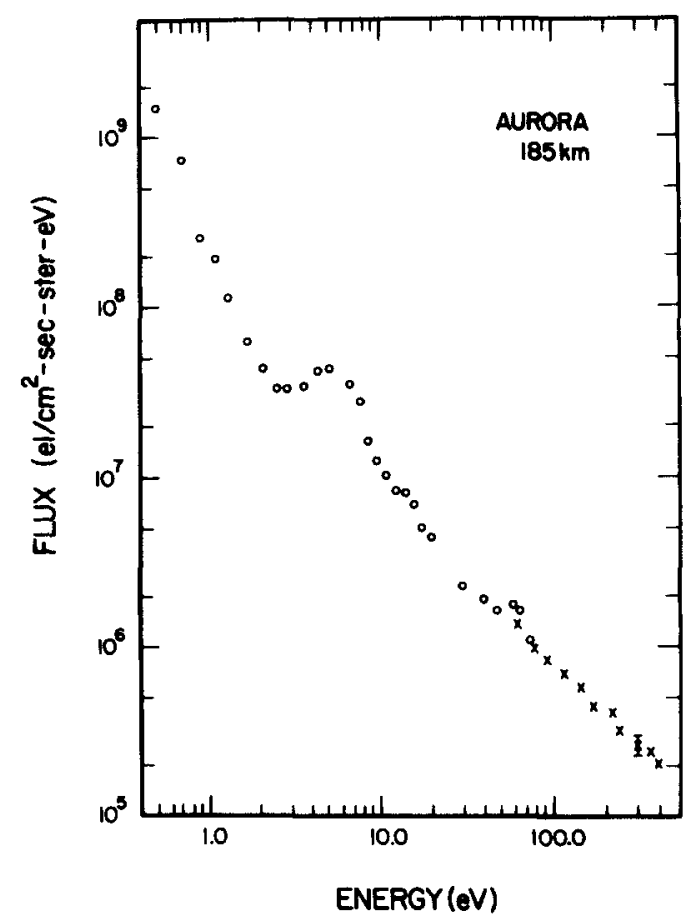

Fig. 17. Low-energy electron flux distribution in the aurora. The open data points were obtained in a low-voltage sweep; the crosses were obtained in a high-voltage sweep.

of instrument entrance aperture, $\Omega=$ solid angle, $\epsilon=$ instrument detection efficiency, and $F=$ particle flux $\left(\mathrm{cm}^{2} \text { sec sr eV) }\right)^{-1}$.

The following assumptions are used to simplify this expression: (a) Particle flux is constant over the sample period; (b) particle flux is isotropic over the instrument field of view; (c) particle flux changes slowly over the energy resolution window; and (d) Channeltron efficiency is constant and near unity from 150 to $650 \mathrm{~V}$ (remember that electrons are accelerated by $150 \mathrm{~V}$ into the Channeltron after leaving the analyzer).

There results, then, the expression

$$
C=\Delta E G\left(E_{0}\right) F\left(E_{0}\right),
$$

where

$$
G\left(E_{0}\right)=\int_{A} d \mathbf{a} \cdot \mathbf{r} \int_{\Omega} d \Omega T\left(E_{0}, a, \Omega\right) \mathrm{cm}^{2} \mathrm{sr},
$$

with $T$ the transmission of the instrument. $G\left(E_{0}\right)=8$ $\times 10^{-4} \mathrm{~cm}^{2}$ sr for the particular geometries of the HARP used on rockets.

Figure 17 illustrates the low-energy auroral electron distribution. It is, in general, typical of low-energy spectral distributions. Features to be noted are the rapid flux increase of the thermal Maxwellian tail below $2 \mathrm{~V}$, the structure in the $1-10 \mathrm{~V}$ region, and the lack of structure above $10 \mathrm{~V}$. The physical processes leading to the shape of the various energy regimes have been discussed in the literature ${ }^{1,22,23}$ in the context of the data. That the magnitude of the flux and its spectral shape are quantitatively correct is given by the fact that energetic electron 
degradation calculations for the atmosphere reproduce the measurements remarkably well. ${ }^{24}$

\section{ACKNOWLEDGMENTS}

This work was supported by NASA Grants NGR-23005-360 and NGR-23-005-015. The authors wish to thank George Carignan for critical reading of the manuscript and Terry Averkamp for the computer program of this work.

'P. B. Hays and A F. Nagy. Planet. Space Sci. 21, 1301 (1973).

2 W. C. Knudsen and G. W. Sharp. J. Geophys. Res. 77, 1221 (1972).

${ }^{3}$ L. A. Frank. N. K. Henderson, and R. L. Swishes, Rev. Sci. Instrum, 40, 685 (1969).

4 P. D. Feldman and J. P. Doering, J. Geophys. Res. 80, 2808 ( 1975 )

${ }^{5}$ P. B. Hays and W. E. Sharp, J. Geophys. Res. 78, 1153 (1973).

"R. Ruedenberg, J. Franklin Inst. 246, 311 (1948)

C. Schmidt. Rev. Sci. Instrum. 41, 117 (1970).
${ }^{*}$ H. D. Zeman, K. Jost, and S. Gilad, Rev. Sci. Instrum. 42, 485 (1971).

" E. Fischer, Z. Phys. (Leipz.) 156, 1 ( 1959).

${ }^{10}$ R. F. Wuerker, H. Stelton, and R.V. Langmuir, J. Appl. Phys. 30, 342 (1959).

$"$ J. A. Simpson, Rev. Sci. Instrum. 32, $128.3(1961)$

${ }^{12}$ W. Steckelmacher, J. Phys. E 6, 1061 (1973).

${ }^{1: 3}$ G. Möllenstedt, Optik 5, 499 (1949).

${ }^{14}$ P. Grivet, Electron Optics (Pergamon, Oxford, 1965), p. 555.

${ }^{15}$ K.-J. Hanszen and R. Lauer, in Focusing of Charged Particles. edited by A. Septier (Academic, New York, 1967).

${ }^{16}$ K. R. Spangenberg. Vacum Tubes (McGraw-Hill. New York. 1948), p. 353 .

${ }^{17}$ T. W. Shyn, R. S. Stolarski, and G. R. Carignan, Phys. Rev. A 6, 1002 (1972)

is A. F. Nagy (private communication).

19 S. E. DeForest, J. Geophys. Res. 77, 651 (1972).

${ }^{20}$ W. E. Sharp (unpublished).

${ }^{21}$ R. A. Basto, W, S. Raitt, and J. J, Sojka. Planet. Space Sci. 24, $115(1976)$

22 W. E. Sharp and P. B. Hays, J. Geophys. Res. 79, 4319 (1974).

${ }^{23}$ W. E. Sharp, Trans. Am. Geophys. Union 56, 1152 (1974).

24 W. E. Sharp, M. H. Rees, A. I. Stewart, and R. A. Hoffman, Trans. Am. Geophys. Union 56, 1032 (1975). 